\title{
TRANSFORMACIONES EN LA FORMACIÓN ACADÉMICA DE LA ESCUELA DE SERVICIO SOCIAL, 1942-1970
}

\section{TRANSFORMATIONS IN THE ACADEMIC EDUCATION OF THE SOCIAL SERVICE SCHOOL, 1942-1970}

\author{
Sonia Angulo Brenes ${ }^{1}$ \\ sanbre.05@gmail.com
}

Fecha de recepción: 6 de junio de 2016 - Fecha de aceptación: 5 setiembre 2016

\begin{abstract}
Resumen
El propósito principal de este artículo es analizar las diferentes transformaciones en la formación académica de la Escuela de Servicio Social en el período de 1942 a 1970; para esto, se revisan las diversas fuentes desde actas del Consejo Universitario hasta los Planes de Estudio; y se examina las tendencias, las rupturas y las continuidades tanto de la formación académica como de los fundamentos del Trabajo Social en su relación con la sociedad costarricense.

La formación ha transmutado constantemente a partir de elementos como el proyecto de creación de la Escuela de Servicio Social propuesto por Héctor Beeche y su respectivo ingreso a la Universidad de Costa Rica, el vínculo con las instituciones relacionadas con la política social, la influencia de los organismos internacionales, el afianzamiento de la relación teoría-práctica, la configuración de los métodos clásicos para la intervención social (propios de Trabajo Social), y el avance al pasar de preparar agentes técnicos a un cuerpo de profesionales formados para atender las manifestaciones de la "cuestión social" en Costa Rica.

Palabras claves: Formación académica, Escuela de Servicio Social, transformaciones, instituciones públicas, organismos internacionales.
\end{abstract}

\begin{abstract}
This article has as objective to analyze the different transformations in the academic education of the Social Service School during the periods from 1942 to 1970. For that, it was necessary to review the sources of information from agreements of the University Council until the study plans and examine the trends, ruptures and continuities of both, the academic education and the bases of the Social Work and their relationship with the Costa Rican society.

The academic education has constantly transmuted from several elements, among them, the project of creation suggested by Hector Beeche and his respective admission to the University of Costa Rica, the link with institutions connected with social policy, the influence of international entities, the consolidation of the theory-practice relationship, the configuration of the traditional methods for the own social intervention of Social Work and the headway to overcome the education of technical agents to a professional bodies formed to attend the manifestations of the "social issue" in Costa Rica.

Key words: Academic education, Social Service School, transformations, public institutions, international entities.
\end{abstract}

1 Escuela de Trabajo Social, Universidad de Costa Rica, Sede Rodrigo Facio. 
El presente artículo surge como resultado de la actividad de investigación denominada "Rupturas y continuidades de los fundamentos sobre la Escuela de Trabajo Social” y posee como objetivo aprehender las principales tendencias, rupturas y continuidades de la formación académica de la Escuela de Servicio Social de la Universidad de Costa Rica, a partir de su articulación con la sociedad costarricense, es decir, lo que se intenta es discutir sobre los elementos de concatenación entre esta formación y la realidad social.

A partir de las fuentes principales -a saber, planes de estudio, informes de la Escuela y actas del Consejo Universitario-, se intenta analizar los cambios en la formación académica de la Escuela de Servicio Social (1942-1970) y tener un primer acercamiento a la relación entre estos y las demandas de la sociedad costarricense para el período en estudio.

De tal manera que antes de discutir y revisar las transformaciones de esta primera expresión educativa, es preciso aclarar y enfatizar que el punto de partida del análisis, se expresa en una comprensión de la formación académica en Trabajo Social:

(...) como un proceso contradictorio, que contiene y entrelaza una serie de elementos filosóficos, históricos, teóricos, metódicos, políticos, éticos, técnicos, culturales, ideológicos, y que se materializan en la lógica de reproducción de la profesión, es decir los fundamentos del Trabajo Social (Angulo, 2014, p. 327).

Esta discusión se fundamenta en cuatro elementos: la formación en sí misma, el vínculo con la universidad pública, lo académico y la formación profesional, tal cual se explica a continuación:

- La formación en su reproducción ontológica, es decir, en las formas que adquiere en el desenvolvimiento de la sociedad, debe ser entendida como una expresión contradictoria; en tanto, en la lógica capitalista, se convierte en un instrumento hacia la preparación para el trabajo, propia del capital y al mismo tiempo en un espacio de la clase trabajadora para apropiarse de la formación de conocimientos que permiten la destrucción del capital (Tonet, 2006).

- Se encuentra entrelazada con la universidad pública como un espacio complejo determinado por las transformaciones histórico-sociales; por lo tanto, como señala Lessa (2006), la producción y la apropiación del conocimiento se encuentran mediados por la sociedad de clases y los procesos sociales más complejos.

- Lo académico se convierte en el vínculo entre la formación y el espacio de la universidad pública, por ende, se encuentra mediado por las contradicciones de la reproducción de la sociedad, se expresa en una lucha por la determinación de un proyecto hegemónico, es decir, no se encuentra libre de contradicciones.

- La formación en Trabajo Social implica comprenderla como profesión a partir de su reproducción histórica; es decir, aprehender el significado de Trabajo Social en la sociedad, intentando superar “(...) el rechazo de la historia, traducido en la búsqueda de entender el Servicio Social en sí y para sí mismo" (Iamamoto, 2000, p. 96).

En síntesis, la formación académica necesita de una serie de conocimientos indisolubles y constitutivos de la profesión que le permiten una lectura, análisis y un vínculo con la sociedad. De tal manera que, para comprender las principales tendencias, continuidades y rupturas de esta en la Escuela de Servicio Social, es necesario superar la visión inmediatista curricular y adentrarse en la comprensión de las relaciones con la sociedad costarricense.

\section{Breve contextualización de las condiciones de vida de la sociedad costarricense entre 1942-1970}

Para entender la reproducción histórica de la sociedad costarricense en el período en estudio, es necesario apuntalar algunos elementos fundamentales que determinaron sus rasgos constitutivos y que 
se entrelazan con las preocupaciones de la formación académica de la Escuela de Servicio Social; las cuales, según Angulo (2014), fueron:

- La expansión de las relaciones capitalistas de producción a partir del café y el azúcar y diversas repercusiones,

- La producción del banano, su conformación como monopolio a partir de la entrada del capital estadounidense,

- Las alteraciones en las condiciones de vida de la clase obrera y el campesinado y su vínculo con propuestas organizativas,

- La semiindustrialización a través del mecanismo de la importación y sus efectos en la economía del país,

- La lógica del Estado y su intervención en las manifestaciones de la cuestión social.

De tal manera, que la constitución del país se entrelaza con las expresiones de un capitalismo internacional, cuyo interés se centró en la dominación de la economía local, principalmente desde el capital estadounidense.

A finales del siglo XIX, según Ramírez y Solís (1979), la concentración de capitales agrícolas fomentó la diferenciación con respecto a los agroindustriales, principalmente el café y el azúcar; lo que permitió la consolidación del mercado interno:

(...) los principales azucareros formaban parte de una oligarquía burguesa dinámica, con tendencias monopólicas alrededor de la cual convergía el capital agrícola, agroindustrial, mercantil, financiero y más tarde el industrial. De ahí que las grandes haciendas azucareras, eran a su vez cafetaleras y contaban respectivamente con su ingenio y su beneficio (Botey y Cisneros, 1981, p.20).

El café se constituyó en el principal cultivo de exportación, lo que generó la dependencia de la economía costarricense y a partir de ahí múltiples repercusiones, tales como la agudización de la concentración de la tierra, la consolidación de una división del trabajo, la precarización de las condiciones de vida del campesinado y su diferenciación con la clase cafetalera oligárquica (Gudmundson, 2010).

Por lo que el café consolidó los primeros atisbos de las relaciones capitalistas de producción; las cuales, se fortalecieron después con el monopolio del banano y la expansión de los transportes, principalmente, por la construcción del ferrocarril al Atlántico. La expansión del banano se extendió a finales del siglo XIX hasta la primera mitad del XX, a partir del afianzamiento de la intervención estadounidense, de tal manera:

(...) la región de Limón se convirtió (...) en el virtual principado del imperio bananero de la United: dueña de las plantaciones, de inmensas cantidades de tierras vírgenes y de todas las líneas de ferrocarril; a ello se sumaban la concesión del manejo de los muelles y el predominio casi absoluto, en cuanto a líneas navieras, de los barcos controlados por la compañía (Pérez, 1997, p.69).

Así, según Carcanholo (1981), entre el período 1950-1967, las exportaciones de banano representaban el 91,8 \% y para 1967, el 60,9\%, este último dato se comprende en función de que la economía del país, a partir de la década del cincuenta, se centró en la expansión de la producción manufacturera y en una incipiente industrialización que se sustentó en el modelo de sustitución de importaciones, apoyándose en dos aspectos: la aprobación de la "Ley de Protección y Desarrollo Industrial (1959) y la incorporación del país al Mercado Común Centroamericano en 1963, de tal manera que:

(...) el Gobierno impulsó el desarrollo del sector manufacturero y su integración a la economía regional centroamericana. Esta estrategia se realizó sin dejar de lado las tradicionales políticas de fomentar la agroexportación, ya que estas generaban los recursos para financiar el crecimiento económico (León y otros, 2014, p.27). 
Todo esto produce la consolidación del proceso de semiindustrialización, el fortalecimiento de un Estado interventor con una inversión en empresas nacionales y el fomento de "monopolios estatales" como la Fábrica Nacional de Licores y el Instituto Nacional de Seguros (León y otros, 2014).

De tal manera, que el Estado se desarrolló en dos vías; por un lado, a través de la inversión de capital en aquellos sectores más productivos; por otro, en el desarrollo de la política social y la creación de una institucionalidad pública que poseían como base la atención a las manifestaciones de la cuestión social, tales como el desempleo, la ausencia de vivienda, la pauperización, entre otros.

Finalmente, un último elemento que se destaca son las alternaciones en las condiciones de vida del campesinado y de la clase obrera y ante esto las expresiones de su organización, así lo manifiesta el Diario de Costa Rica en 1942 “(...) el empobrecimiento cada día mayor de nuestros campesinos, [lo que] puede ocasionar en cualquier momento, no sólo la protesta airada de las masas, sino también el derrumbe de la vitalidad de nuestro pueblo" (p. 1).

Todo esto reflejó la agudización de las condiciones de vida de las clases subalternas costarricenses frente a la expansión de un capital nacional e internacional que propició las bases para la consolidación de una Costa Rica más desigual.

Así, para la década del sesenta, se intensifica el desempleo, la situación de la precarización de la vivienda, el aumento de la mortalidad infantil y la deserción educativa; lo que generó la proliferación de una serie de instituciones que responden a estos escenarios y que produce el aumento de empleados públicos (Aguilar, 1989).

De tal manera que todos estos rasgos socio-históricos consolidaron el surgimiento de la profesión de Trabajo Social en Costa Rica, en la década de 1940, tal como lo indica Esquivel (2003):

(...) las condiciones básicas que llevan a la génesis del Trabajo Social en Costa Rica son la coyuntura de la crisis liberal y los efectos de la transformación del capitalismo mundial que toma la orientación monopólica, las presiones de la sindicalización como respuesta a la explotación capitalista y un empobrecimiento extremo de la mayoría de la población nacional (p.111).

En síntesis, la conformación de la profesión se enlaza con la intervención de las manifestaciones de la cuestión social y con su concatenación con la política social, de ahí que sus preocupaciones en la formación académica se expresen en un vínculo estrecho entre el Estado, su institucionalidad y la Escuela de Servicio Social, tal cual se presenta en el siguiente apartado.

\section{Continuidades y rupturas de la formación académica de la Escuela de Servicio Social}

La formación académica de la Escuela de Servicio Social a lo largo de su historia ha conllevado diferentes tendencias que entrelazan continuidades y rupturas; las cuales han expresado y reflejado su relación con la sociedad costarricense, a partir de todos los cambios acaecidos en el contexto histórico expuesto.

Esto se expresa en los Planes de Estudio y las formas que se han ido retratando en diferentes momentos de la formación académica; así, según Esquivel (2007), “(...) la madurez de la formación profesional en Trabajo Social, se ha ido forjando entre procesos con intentos de ruptura, impulsada por una fuerte orientación de dar respuesta a las demandas del contexto nacional y latinoamericano" (p.74).

En este sentido, en el surgimiento y la reproducción de la formación académica de la Escuela de Servicio Social, se puede identificar tendencias; en las cuales, se expresan intentos de ruptura y continuidades tales como:

- Condiciones de vida de la sociedad costarricense, tratamiento de la cuestión social y surgimiento de la Escuela de Servicio Social,

- El posterior ingreso a la Facultad de Ciencias Económicas y Sociales como expresión de vínculo con la Universidad de Costa Rica, 
- El contexto imperialista de Estados Unidos en América Latina y su reflejo en la formación académica,

- La consolidación de los organismos internacionales y regionales, especialmente la Organización de las Naciones Unidas (ONU) y Organización de los Estados Americanos (OEA),

- El establecimiento de la "práctica académica" como preocupación de la relación teoría y práctica,

Estas expresiones reflejan las formas en que se ha configurado la formación académica y sus concatenaciones con la sociedad costarricense, tales como el vínculo con el campesinado en medio de una economía fundamentalmente agrícola o las reconfiguraciones de la clase obrera en la primera mitad del siglo XX. De modo que las determinaciones históricas en el capitalismo local e internacional discutidas anteriormente impactaron en el surgimiento de la Escuela y su reproducción.

Así, el surgimiento de la Escuela de Servicio Social se establece en el año de 1942, con un carácter privado y propuesto por el abogado Héctor Beeche ${ }^{2}$, en el contexto de una sociedad costarricense con fuertes rasgos de una economía agrícola y, conformada por el campesinado y la formación de la clase obrera, de tal manera que “(...) la Escuela de Servicio Social se arraiga en una sociedad costarricense, que presenta para ese momento, manifestaciones de la "cuestión social” particulares, con una economía basada en el modelo agroexportador y con una incipiente industrialización” (Angulo, 2014, p.217).

La propuesta de Beeche para la creación de la Escuela proponía el vínculo con varias entidades estatales, como el Despacho de Educación Pública, la Universidad de Costa Rica, la Secretaría de Salubridad Pública, el Patronato Nacional de la Infancia y la Caja Costarricense del Seguro Social, de las cuales pretendía lograr su apoyo y financiamiento (Beeche, 1942a, Luján, 1942; Sánchez, 1942 y Padilla, 1942).

A partir de esta propuesta, se concretiza la anuencia para su creación por parte de Alfredo Sánchez, presidente del Patronato Nacional de la Infancia, Guillermo Padilla Castro, subgerente de la Caja Costarricense de Seguro Social y Mario Luján Fernández de la Secretaría de Salubridad Pública, este último expone:

He tenido placer en estudiar con detenimiento el plan presentado por el Licenciado Héctor Beeche, para fundar la Escuela de Servicio Social, como uno de los tantos organismos de carácter universitario. La idea, en mi concepto, tiene la ventaja efectiva de poder preparar por organismos nacionales, elementos idóneos y capacitados, no sólo para el servicio de Protección Social a mi cargo, sino para la Caja del Seguro Social y otras Dependencias (Luján, 1942, p.1).

Como se puede vislumbrar, el surgimiento de la Escuela se encuentra concatenado con el desarrollo de las instituciones de seguridad social y que responden a través de la política respectiva a las manifestaciones de la cuestión social, por lo tanto, su reproducción en los años posteriores responderá a esta lógica.

Aunado a la propuesta de Héctor Beeche de la necesidad de que la Escuela sea parte de la incipiente Universidad de Costa Rica y por esto para 1942 solicita la posibilidad de su ingreso:

\footnotetext{
Es mi creencia que una Escuela que preste servicios eminentemente prácticos y prepare profesionales para una carrera también práctica, estaría destinada a contribuir al engrandecimiento de la Universidad y al mejor cumplimiento de los fines culturales que animaron a Ud., como Secretario de Educación Pública que ha sido el gran sostenedor y campeón de la idea universitaria, y al señor Presidente, al crear la Universidad y al darle rentas, local, vida activa y propulsar sus actuaciones en forma decidida. La Escuela de Servicio Social, como plantel universitario, vendría, creo yo, a cumplir el indicado propósito (Beeche, 1942a, p. 7 y 8).
}

2 Héctor Beeche realizó su formación en Estados Unidos, y según comenta, en esos estudios se dedicó “(...) con preferencia al estudio de la ciencia penitenciaria y a investigaciones sobre previsión social. Tales actividades científicas fueron prohijadas por el Bureau of Prisons del Departamento de Justicia, y por el Children's Bureau, del Departamento del Trabajo, ambas oficinas del Gobierno Federal" (Beeche, 1942a, p. 1). 
En este mismo contexto, en 1942, el ministro de Salubridad solicita al de Educación la posibilidad de ingreso de la Escuela a la Universidad, específicamente a la "Escuela de Ciencias Político-Sociales y Económicas" que se encontraba pronta a fundarse, exponiendo las ventajas de su creación. Así en la Sesión del Consejo Universitario celebrada el 10 de marzo de 1942, se expresa lo siguiente:

(...) El Sr. Zeledón [Representante estudiantil) expone que habiendo conversado con el Sr. Ministro de Salubridad (...) dicho funcionario le ofreció, en el caso de creación de esta Escuela la suma de $₫ 300$ mensuales como una ayuda del Ministerio a la Universidad, prometiendo conseguir la ayuda pecuniaria de la Caja del Seguro Social" (Consejo Académico, 1942, Art. 11).

En 1943, la Escuela de Servicio Social manifiesta su deseo de incorporarse a la Universidad y a la recién fundada Facultad de Ciencias Económicas y Sociales (Consejo Universitario, 1943); posteriormente, el 07 de marzo de 1944, se acuerda la incorporación de la Escuela de Servicio Social a la Universidad de Costa Rica (Consejo Universitario, 1944), como Escuela anexa, así según Angulo (2014):

(...) los primeros atisbos de creación de la Escuela de Servicio Social se vinculan con la posibilidad de incorporarse a la Universidad, con una propuesta de formación eminentemente práctica y con la finalidad de legitimar la utilidad de la profesión en las instituciones (p.219).

De tal manera, que esta primera concreción de la formación académica posee una concatenación con la sociedad costarricense, a través de las instituciones que atienden lo social y que se convierten en espacios fundamentales de influencia en los planes de estudio, de reclutamiento de alumnos, de financiamiento y como espacios sociolaborales. Así lo señala Beeche (1942a):

(...) crear un centro docente para la preparación de personal técnico apto para actuar en los departamentos del Patronato Nacional de la Infancia, de la Caja de Seguros Sociales, de un Tribunal de Menores, del Departamento de Prisiones, de la Secretaría del Trabajo y Previsión Social, de la Secretaría de Salubridad Pública en los ramos de Asistencia Pública, Higiene y Protección Social y Visitaduría Sanitaria, etc., de la Secretaría de Educación Pública en sus ramos de educación especial, deserción escolar, niños retrasados y demás por el estilo; y de dotar a la Universidad de una Escuela que procure a sus alumnos una profesión eminentemente práctica, moderna y útil; con ese afán es que vengo a proponer a Ud. la creación de la Escuela de Servicio Social en Costa Rica, como dependencia de la Universidad (p.8).

Por lo que la propuesta académica de la Escuela en los primeros años se encuentra vinculada a elementos que fomentaron los vínculos con las instituciones mencionadas y que permitieron la constitución de estudiantes en Trabajo Social con conocimientos técnicos en elementos específicos como: higiene y asistencia social, derecho de trabajo y seguro, higiene mental y previsión social, entre otros; todo esto se puede vislumbrar tanto en los cursos preparatorios (desde su creación con carácter privado) como en su primer plan de estudio.

En este sentido, los estudios preparatorios propuestos por Héctor Beeche consistían en un conjunto de cursos que debían llevar las personas antes de iniciar y proseguir con el Plan de Estudio, estos se constituían en los siguientes:

a) Elementos de Derecho de Trabajo y Seguro Social (asignatura diaria).

b) Sociología y Elementos de Estadística (asignatura diaria).

c) Elementos de Higiene y Asistencia Social (asignatura alterna).

d) Elementos de Psicología Social (asignatura alterna) (Beeche, 1942, p.13).

Estos cursos que se constituían en la base preparatoria contenían elementos necesarios para la intervención en las instituciones tales como la Caja Costarricense del Seguro Social y el Patronato Nacional de la Infancia; además, presentaban un interés por elementos de discusión de aspectos vinculados con el Derecho, la Sociología, la Estadística y la Psicología. 
Posteriormente, estos cursos se constituyeron en la base del primer Plan de Estudio de la Escuela, cuyo fundamento se estableció a partir de la "American Association of Schools of Social Work", en 1932 y que proponía cuatro años de formación. La malla curricular fue discutida por los representantes de las instituciones públicas; Mario Luján Fernández, Guillermo Padilla Castro, Antonio Bruck, Alfredo Sánchez, Oscar Barahona Streber (Jefe de la Sección de Propaganda y Educación Social de la Caja de Seguro Social) y finalmente Abel Guier Alvarado (Beeche, 1942a y 1942b). En el Cuadro 1, se presenta la propuesta.

Cuadro 1

Propuesta de primer Plan de Estudios de la Escuela de Servicio Social, realizada por Héctor Beeche, 1942

\begin{tabular}{|c|c|}
\hline Niveles & Cursos \\
\hline Primero & $\begin{array}{l}\text { 1. Prolegómenos del Derecho General y del Trabajo. } \\
\text { 2. Anatomía y Fisiología generales. } \\
\text { 3. Psicología General. } \\
\text { 4. Derecho Penal Elemental. } \\
\text { 5. Higiene Social ( } 1^{\text {er }} \text { curso). }\end{array}$ \\
\hline Segundo & $\begin{array}{l}\text { 1. Higiene Social ( } 2^{\text {do }} \text { curso). } \\
\text { 2. Sociología general y aplicada. } \\
\text { 3. Servicio Social ( } 1^{\text {er }} \text { curso). } \\
\text { 4. Higiene mental y Psiquiatría Social. } \\
\text { 5. Psicología aplicada. }\end{array}$ \\
\hline Tercero & $\begin{array}{l}\text { 1. Derecho del Trabajo. } \\
\text { 2. Servicio Social ( } 2^{\text {do }} \text { curso). } \\
\text { 3. Economía doméstica. } \\
\text { 4. Pedagogía correctiva. } \\
\text { 5. Delincuencia juvenil. }\end{array}$ \\
\hline Cuarto & $\begin{array}{l}\text { 1. Servicio Social ( } 3^{\text {er }} \text { curso). } \\
\text { 2. Seguro Social. } \\
\text { 3. Estadística Social. } \\
\text { 4. Previsión Social Administrativa. } \\
\text { 5. Asistencia Pública. }\end{array}$ \\
\hline
\end{tabular}

Fuente: Angulo (2014), de acuerdo con Beeche, 1942b, p. 2 y 3.

Es importante exponer que esta primera propuesta no logró concretarse, debido a varias razones; por un lado, las condiciones socio-históricas de Costa Rica no coincidían con ella, pues la malla curricular poseía como antecedente principal la oferta norteamericana. Por otro lado, el peso de la influencia de las entidades públicas incidió en el establecimiento de otras discusiones de interés para estas en la oferta formativa.

Este último aspecto se refleja en los fines de la Escuela de Servicio Social en el año 1942, en donde, se busca fortalecer la influencia de las instituciones encargadas de la política social en la formación académica, en donde se expresa lo siguiente:

La Escuela de Servicio Social pretende formar personas técnicamente preparadas para contribuir al mejoramiento y progreso del pueblo costarricense, objetivo que logrará con el esfuerzo de sus organizadores, y muy particularmente, con la colaboración de entidades y especialistas extranjeros que, en su oportunidad, se tratará de hacer llegar al país (Beeche, 1942, p.13). 
Tal cual se refleja en los fines planteados, se espera que en la formación académica no solo se logre un cuerpo de agentes técnicos con un adiestramiento práctico, sino también que desde las instituciones puedan influenciar en esta propuesta; en estos mismos propósitos, se presenta la necesidad de la intervención de organismos internacionales que luego analizarán la formación, tales como la Organización de las Naciones Unidas (ONU) y la Organización de los Estados Americanos (OEA).

Aunado a la influencia de la Facultad de Ciencias Económicas y Sociales, en donde la Escuela en 1947 se constituía en "Sección de Servicio Social" (Consejo Universitario, 1946 y 1947); posteriormente, en 1952, en "Sección de Sociología y Servicio Social" (Consejo Universitario, 1952) y; finalmente, se consolida de 1953 hasta 1955 como Departamento (Consejo Universitario, 1953 y Escuela de Servicio Social, 1955).

En síntesis, estas tres primeras tendencias de la Escuela de Servicio Social permitieron definir varios aspectos;

1. La constitución de un cuerpo de agentes técnicos que necesitaba la emergente institucionalidad pública y su influencia en su formación académica vinculado a la propuesta de un Estado reformista conservador,

2. La influencia de la Universidad de Costa Rica desde la propuesta de la Facultad de Ciencias Económicas y Sociales y

3. Las primeras expresiones de vínculo con los organismos internacionales.

Esta última se va fortalecer debido a varias causas; dos ellas son: el contexto imperialista de los Estados Unidos y su incidencia en la formación académica en Trabajo Social a través de los estudios de la Organización de las Naciones Unidas (ONU), en donde se discutía recomendaciones para la formación en los países de América Latina, y; el ingreso de Francisco Herrera como director de la Escuela de Servicio Social en 1948. Según Angulo (2014):

Es en esta lógica que se establece una formación académica con fuerte influencia de Estados Unidos, y con algunos elementos de los países de América del Sur, fuertemente imbricada con los intereses de organizaciones como la Organización de las Naciones Unidas (ONU) y la Organización de los Estados Americanos (OEA) (p.223).

Esta intervención de los organismos internacionales se consolida en la Escuela de Servicio Social, principalmente, en los años cincuenta y sesenta cuando ingresan dos asesores de las Naciones Unidas que revisan la malla curricular y proponen cambios fundamentales:

- El primero es la consolidación de la práctica académica en el Plan de Estudio de 1954, propuesto por la asesora Guillermina Llanusa ${ }^{3}$.

- El segundo la reforma del Plan de Estudio de 1963 a 1964 propuesto por el asesor Jean Labbens.

El primer elemento refiere a la instauración de la práctica académica en la formación académica, la cual se constituía en el camino propicio para el vínculo con la sociedad costarricense, a través de su realización en instancias como las instituciones públicas; de modo que en los primeros momentos

3 La Dra. Guillermina Llanusa y Montes fue asesora en Bienestar Social de las Naciones Unidas, colaboró con la Escuela de Servicio Social, en el período de 1953-1954 (Consejo Universitario, 1953a). Además, impartió un curso especial de Supervisión, en marzo de 1953 (Consejo Universitario, 1953b). 
“(...) la práctica se lleva a cabo durante los tres años de formación, en agencias privadas e instituciones públicas, bajo el cuidado de profesores expertos” (Universidad de Costa Rica, 1960).

La práctica se instaura en el Plan de Estudio de 1954, en cada uno de sus niveles, y en diferentes ámbitos, tales como Estudio de Casos en Familias y Niños, Trabajo Social con Grupos, Servicio Social Criminológico y Trabajo Social Médico, que se establecen como elementos propios de la configuración de las instituciones sociales para ese momento. Esto se refleja en el Cuadro 2, donde se expone el Plan de Estudio para ese año:

Cuadro 2

Plan de Estudio 1954 y la concreción de la práctica académica

\begin{tabular}{|c|c|}
\hline Niveles & Cursos \\
\hline Primer año & $\begin{array}{l}\text { Principios de Economía, Principios de Sociología, Principios de Administración, Administración } \\
\text { Pública, Historia Económica y Social General, Introducción al Servicio Social (a) Historia del } \\
\text { Bienestar Social; b) Trabajo Social Individual. } \\
\text { Práctica Primer semestre: Visitas a instituciones. Segundo semestre: Estudio de Casos. }\end{array}$ \\
\hline Segundo año & $\begin{array}{l}\text { Métodos del Servicio Social (Trabajo Social Individual y Trabajo Social con Grupos), Bienestar de } \\
\text { la Familia, Psicología General, Derecho Positivo y Servicio Social, Sindicatos y Legislación Social, } \\
\text { Estadística General. Práctica Primer semestre: Estudio de Casos de Familias y Niños. Segundo } \\
\text { semestre: Trabajo Social con Grupos. }\end{array}$ \\
\hline Tercer año & $\begin{array}{l}\text { Sociología Rural y Urbana, Organización de la Comunidad, Psicología Aplicada, Psiquiatría, Higiene } \\
\text { Mental, Medicina Social y Nutrición, Criminología, Penología. } \\
\text { Práctica Primer semestre: Servicio Social Criminológico. Segundo semestre: Trabajo Médico } \\
\text { Social. }\end{array}$ \\
\hline \multicolumn{2}{|c|}{$\begin{array}{l}\text { Con la aprobación de estos tres años “(...) se extendía un diploma de Conclusión de Estudios de Servicio Social” (Escuela } \\
\text { de Servicio Social, 1955, p. 105) }\end{array}$} \\
\hline $\begin{array}{l}\text { Para la obtención de } \\
\text { la Licenciatura }\end{array}$ & $\begin{array}{l}\text { Se deben aprobar las siguientes asignaturas en el campo de Ciencias Económicas y Sociales: } \\
\text { Administración de Instituciones de Servicio Social, Cooperativas, Estudio de Expedientes Sociales, } \\
\text { Historia Económica y Social de Costa Rica, Investigación Social, Doctrinas Económicas, Ingreso } \\
\text { Nacional, Derecho de Trabajo, Teoría de la Competencia, Seguridad Social, Teoría de la Ocupación } \\
\text { y Problemas Sociales Contemporáneos. Asimismo, presentar un examen de grado y la presentación } \\
\text { de una tesis ante la Facultad. }\end{array}$ \\
\hline
\end{tabular}

Fuente: Elaboración propia, de acuerdo con Escuela de Servicio Social, 1954 y 1955, p. 105 y 106.

En este sentido, la práctica académica se constituía como un intento de ruptura en la formación académica, pues era la forma en que el estudiantado se vinculaba con la sociedad costarricense y, al mismo tiempo, se expresaba como una continuidad, pues esa concatenación se establecía desde una mirada concreta, es decir, desde el trabajo con las instituciones.

El otro intento de ruptura en la formación se establece en el año 1964, a partir de la segunda intervención de las Naciones Unidas en la Escuela de Servicio Social desde la lectura del asesor el Dr. Jean Labbens, el cual en su estudio propuso varias recomendaciones que implicaban cambios profundos, entre ellos la reforma del Plan de Estudio. Este análisis discutió la necesidad de que la formación académica lograra un mayor posicionamiento con respecto a la sociedad costarricense, tal cual se expone: 
La primera impresión que recoge un observador es que el Servicio Social no ha tomado todavía en Costa Rica la importancia que merece y que está llamado a tomar dentro de las perspectivas del desarrollo económico y social. Por una parte el número de trabajadores sociales, sobre todo de los titulados, es todavía muy reducido; por otra parte, la profesión no recibe ni la remuneración ni el prestigio que le correspondría [sic]. [Además agrega sobre la formación] La organización de la escuela refleja, ella también, esta situación: (...) parece tender sobre todo a profesionalizar trabajadores que recibirían, sobre el terreno y de modo empírico lo esencial de su formación (Labbens, 1964, p.1).

Asimismo, Labbens (1964) realiza una crítica a la formación académica de la Escuela de Servicio Social, expresando que su proyecto profesional es limitado y que no logra configurarse en un proyecto académico, sino más bien se ha constituido en un complemento de formación, es decir, en una preparación técnica; así lo expone:

(...) la escuela funciona menos como una escuela verdadera que como un centro de profesionalización para personas que han ingresado en el Servicio Social antes de haber recibido una formación correspondiente. Pero la palabra profesionalización es inexacta por su exceso de optimismo; el número de trabajadores sociales titulados que salen de la escuela casi es insignificante. No se trata pues de una verdadera profesionalización, sino más bien de un complemento de formación (o de información) distribuido a "empíricos" que no necesitan verdaderamente un diploma para conservar su oficio y hacer carrera (p.7).

A partir de esta crítica, la propuesta de Labbens (1964) se centra en cuatro líneas, en donde, se propone la fundamentación de la reforma del Plan de Estudios, tal cual se presenta en el Cuadro 3:

\section{Cuadro 3}

Propuesta de proyecto de formación de la Escuela de Servicio Social por Jean Labbens, 1964

\begin{tabular}{|c|c|c|c|c|}
\hline Línea & $\begin{array}{l}\text { Vínculo con las } \\
\text { Ciencias Sociales }\end{array}$ & $\begin{array}{l}\text { Énfasis en el } \\
\text { Trabajo Social }\end{array}$ & Cursos optativos & Prácticas intensivas \\
\hline Propuesta & $\begin{array}{l}\text { A través de cursos y } \\
\text { seminarios: } \\
\text { a) Psicología Social y } \\
\text { Sociología de las desvia- } \\
\text { ciones. } \\
\text { b) Estructura social y } \\
\text { Trabajo Social. } \\
\text { c) Ciencia y Práctica en } \\
\text { el Trabajo Social. }\end{array}$ & $\begin{array}{l}\text { Por medio de: } \\
\text { a) Casework. } \\
\text { b) Groupwork. } \\
\text { c) Desarrollo de la comu- } \\
\text { nidad. } \\
\text { d) Organización de } \\
\text { los servicios sociales: } \\
\text { administración de los } \\
\text { programas. } \\
\text { e) Filosofía e historia del } \\
\text { Trabajo Social. }\end{array}$ & $\begin{array}{l}\text { El paso de un cuerpo } \\
\text { de cursos obligatorios } \\
\text { a optativos, entre ellos } \\
\text { Sociología Rural y } \\
\text { Urbana. }\end{array}$ & $\begin{array}{l}\text { Centradas en los métodos } \\
\text { del Servicio Social: } \\
\text { a) Primer año: Casework } \\
\text { y Groupwork. } \\
\text { b) Segundo año: } \\
\text { Desarrollo de la comu- } \\
\text { nidad. }\end{array}$ \\
\hline
\end{tabular}

Fuente: Angulo, 2014, de acuerdo con Labbens, 1964, págs. 17-18.

La asesoría de Jean Labbens se constituyó en un cambio fundamental, en tanto, es a partir de su estudio que se expresaron modificaciones fundamentales en la formación académica, tales como el vínculo con las Ciencias Sociales, la consolidación de la trilogía metódica ${ }^{4}$, especialmente, la entrada del "Desarrollo de la Comunidad" y la consolidación de la lógica de la práctica académica, cambios que se expresaron en el Plan de Estudio de 1965.

4 Consistía en la inclusión de los tres métodos tradicionales de Trabajo Social propios de la influencia de la formación norteamericana en las Escuelas de Servicio Social latinoamericanas, a saber: método de caso o casework (1917), el de grupo o groupwork (1946) y finalmente la organización de la comunidad (1947) (Torres, 2006). 
En este sentido, las preocupaciones que expusieron tanto Guillermina Llanusa como Jean Labbens impactaron en la formación académica y produjeron cambios, principalmente, en los Planes de Estudio y en el vínculo de la Escuela con la sociedad.

Por lo tanto, tanto la propuesta de la consolidación de la práctica académica como la reforma del Plan de Estudio de 1964 permitieron reforzar la preocupación de la formación académica por la relación teoría-práctica, tratando de formar un cuerpo de profesionales preocupado por este vínculo en función de la concatenación con la sociedad costarricense, así en 1968 se logra expresar la centralidad en este elemento y en el impulso de los métodos de caso, grupo y comunidad; tal cual se refleja en los fines:

a) Dar al estudiante las bases teóricas necesarias para una mejor comprensión de los fenómenos de la vida social y para la promoción del bienestar de los individuos, los grupos y la comunidad.

b) Dar al estudiante la suficiente oportunidad práctica necesaria para que se aplique sus conocimientos teóricos, desarrolle una actividad profesional en el trabajo, y conozca la comunidad en que se desenvuelve (Escuela de Servicio Social, 1968, p. 6).

La preocupación por la relación teoría-práctica para la concreción del Plan de Estudio de 1968 se configura en una ruptura con respecto a los primeros años de creación y desarrollo de la Escuela de Servicio Social, pues se le otorga un tratamiento especial.

Finalmente, una continuidad que se encuentra a lo largo del período estudiado es la preocupación por el vínculo con la sociedad costarricense a través de la institucionalidad pública vinculada a la atención de las manifestaciones de la "cuestión social" desde las políticas sociales. Este aspecto se refleja en los fines de la Escuela, la práctica académica, la conformación de los planes de estudio y la influencia ejercida por estas en la formación académica en general.

\section{Conclusiones}

A partir de esta somera reconstrucción histórica de la formación académica de la Escuela de Servicio Social, se puede entender sus transformaciones; por un lado, en su surgimiento, la intencionalidad del proyecto de Héctor Beeche y el ingreso a la Universidad de Costa Rica; y por otro, en su reproducción, la inquietud constante por el vínculo con la sociedad costarricense a través de las instituciones públicas y la influencia de los organismos internacionales, lo cual estableció diferentes concatenaciones con la sociedad costarricense.

Por lo que, a lo largo de las transformaciones de la formación académica de la Escuela, se pueden entrever diversos intentos de ruptura en términos de sus fundamentos; los cuales no logran la superación de la base conservadora de la profesión ni su carácter de intervención, de ahí su constante preocupación por la relación con las instituciones vinculadas a la política social.

Asimismo, estos intentos de ruptura se expresaron a partir de la influencia ejercida por los organismos internacionales, especialmente por las Naciones Unidas a través de sus asesores; quienes, a partir de sus recomendaciones, influenciaban los cambios realizados en los Planes de Estudio; ejemplo de esto se refleja en la propuesta formativa de 1954 y 1965.

La centralidad de las instituciones en la formación académica se configura como una expresión particular en la Escuela de Servicio Social de la Universidad de Costa Rica, pues esta relación influenció los Planes de Estudio, el financiamiento, el vínculo con los estudiantes y posteriormente la concatenación con la sociedad costarricense a través de la práctica académica. Este último vínculo consolidó la preocupación por la relación teoría-práctica y se concretó en los vínculos con la realidad social.

Por lo tanto, las tendencias, intentos de ruptura y las continuidades repercuten en la lógica de una formación académica conservadora, con fuertes rasgos de influencia norteamericana, con planteamientos 
positivistas, funcionalistas y pragmatistas que no permitieron rupturas fundamentales en el período de estudio, propiamente en la reproducción de los fundamentos de la Escuela de Servicio Social.

\section{Bibliografía}

Aguilar, Marielos. (1989). Clase trabajadora y organización sindical en Costa Rica: 1943-1971. San José, Costa Rica: Editorial Porvenir-FLACSO-ICES.

Angulo, Sonia. (2014). Fundamentos de la complejidad agraria-campesina y rural en la formación académica de la Escuela de Servicio Social de la Universidad de Costa Rica, 1940-1970 (Tesis de maestría sin publicar). Universidad de Costa Rica, Costa Rica.

Beeche, Héctor. (31 de marzo de 1942). La Escuela de Servicio Social. Diario de Costa Rica. pág. 13.

Beeche, Héctor. (1942a). Solicitud de fundación de la Escuela de Servicio Social. Correspondencia enviada al Secretario de Estado en el Despacho de Educación Pública. Recuperado de Archivo Nacional. Día 26 de noviembre del 2012.

Beeche, Héctor. (1942b). Plan de Estudios y programa de la “Escuela de Servicio Social”. Correspondencia enviada al Secretario de Estado en el Despacho de Educación Pública. Recuperado de Archivo Nacional. Día. 26 de noviembre del 2012.

Botey, Ana. y Cisneros, Rodolfo. (1981). La fundación del Partido Comunista de Costa Rica. (Tesis de Licenciatura sin publicar). Universidad de Costa Rica, Costa Rica.

Carcanholo, Reinaldo. (1981). El desarrollo del capitalismo en Costa Rica. San José, Costa Rica: Editorial Universitaria Centroamérica (EDUCA).

Consejo Universitario. (10 de marzo de 1942). Acta de la Sesión Ordinaria № 004 del Consejo Universitario. Artículo 11. Recuperado del Archivo del Consejo Universitario. Día 10 de julio de 2013.

Consejo Universitario. (30 de noviembre de 1943). Acta de la Sesión Ordinaria $\mathrm{N}^{\circ} 030$ del Consejo Universitario. Artículo 13. Recuperado del Archivo del Consejo Universitario. Día 10 de julio de 2013.

Consejo Universitario. (07 de marzo de 1944). Acta de la Sesión Ordinaria N002 del Consejo Universitario. Artículo 14. Recuperado del Archivo del Consejo Universitario. Día 10 de julio de 2013.

Consejo Universitario. (17 de diciembre de 1946). Acta de la Sesión Extraordinaria $\mathrm{N}^{\circ} 048$ del Consejo Universitario. Artículo 06. Recuperado del Archivo del Consejo Universitario. Día 10 de julio de 2013.

Consejo Universitario. (18 de febrero de 1947). Acta de la Sesión Extraordinaria N E03 del Consejo Universitario. Artículo 10. Recuperado del Archivo del Consejo Universitario. Día 10 de julio de 2013. 377

Consejo Universitario. (01 de diciembre de 1952). Acta de la Sesión Ordinaria Nº51 del Consejo Universitario. Artículo 08. Recuperado del Archivo del Consejo Universitario. Día 10 de julio de 2013.

Consejo Universitario. (02 de marzo de 1953). Acta de la Sesión Ordinaria $\mathrm{N}^{\circ} 061$ del Consejo Universitario. Artículo 53. Recuperado del Archivo del Consejo Universitario. Día 10 de julio de 2013.

Consejo Universitario. (31 de agosto de 1953a). Acta de la Sesión Ordinaria N ${ }^{\circ} 87$ del Consejo Universitario. Artículo 14. Recuperado del Archivo del Consejo Universitario. Día 10 de julio de 2013.

Consejo Universitario. (23 de noviembre de 1953b). Acta de la Sesión Ordinaria $N^{\circ} 100$ del Consejo Universitario. Artículo 10. Recuperado del Archivo del Consejo Universitario. Día 28 de mayo de 2013.

Diario de Costa Rica. (09 de enero de 1942). Existe un estado manifiesto de miseria y de injusticia social. Diario de Costa Rica. pp 1 y 6.

Escuela de Servicio Social. (1954). Plan de Estudios de la Escuela de Servicio Social 1954. Recuperado de Escuela de Trabajo Social. Día 18 de julio del 2013. 
Escuela de Servicio Social. (1955). Plan de Estudios de la Escuela de Servicio Social 1955. Recuperado de Escuela de Trabajo Social. Día 18 de julio del 2013.

Escuela de Servicio Social. (1968). Programa de Trabajo. Cátedra de Práctica Supervisada (SS-212). Escuela de Servicio Social. Recuperado de Archivo Universitario. Folder 9878. Día 27 de marzo de 2012.

Esquivel, Freddy. (2007). Notas sobre la formación en la Escuela de Trabajo Social, Universidad de Costa Rica (UCR) durante las décadas de 1950 a 1980. Revista Educación. 31 (2). 59-78.

Esquivel, Freddy. (2003). Génesis y reproducción del Trabajo Social en Costa Rica (Tesis de maestría). Universidad de Costa Rica, Costa Rica.

Gudmundson, Lowell. (2010). Costa Rica antes del café. San José, Costa Rica: Editorial Universidad Estatal a Distancia (EUNED).

Iamamoto, Marilda. (2000). La metodología en el Servicio Social: lineamientos para el debate. En E. Borgianni y C. Montaño (Orgs), Metodología y Servicio Social: Hoy en debate. (pp. 93-104). São Paulo. Brasil: Cortez Editora.

Labbens, Jean. (1964). Informe sobre la Escuela de Servicio Social de la Universidad de Costa Rica. Escuela de Servicio Social. Recuperado de Archivo Universitario. Folder 9873. Día 13 de marzo de 2012.

León, Jorge y otros. (2014). Historia económica de Costa Rica en el siglo XX: Crecimiento y las políticas económicas. Tomo I. San José, Costa Rica: Editorial de la Universidad de Costa Rica.

Lessa, Sergio. (2006). Universidade: produção e apropriação do conhecimento. Recuperado de: http:// www.sergiolessa.com/no_published/universidade_conhecimento.pdf. Día: 30/08/2013.

Luján, Mario. (19 de febrero de 1942). Correspondencia enviada a Luis Demetrio Tinoco. Presidente del Consejo Universitario. Número 7-a-454. Recuperado de Archivo Nacional. Día 26 de noviembre de 2012.

Padilla, Guillermo. (25 de febrero de 1942). Correspondencia enviada a Luis Demetrio Tinoco. Presidente del Consejo Universitario. Recuperado de Archivo Nacional. Día 26 de noviembre de 2012.

Pérez, Héctor. (1997). Breve historia contemporánea de Costa Rica. México. Fondo de Cultura Económica.

Ramírez, Mario y Solís, Manuel. (1979). El desarrollo capitalista en la industria costarricense (18501930) (Tesis de Licenciatura sin publicar). Universidad de Costa Rica, Costa Rica.

Sánchez, Alfredo. (25 de febrero de 1942). Correspondencia enviada a Luis Demetrio Tinoco. Presidente del Consejo Universitario. Recuperado de Archivo Nacional. Día 14 de marzo de 2013.

Tonet, Ivo. (2006). Educação e Formação Humana. En: http://www.ivotonet.xpg.com.br/. Día 25/03/2013, Hora: 5:30 p.m.

Torres, Jorge. (2006). Historia del Trabajo Social. Buenos Aires. Argentina: Editorial Lumen Hvmanitas.

Universidad de Costa Rica. (1960). Planes de estudios y requisitos: 1961-1962. San José, Costa Rica: Universidad de Costa Rica. 
